\title{
Patient safety: time for a radical re-think
}

\section{DOI: $10.7861 /$ fhj.ed.8.3.2}

The theme of patient safety is particularly topical, given the current climate in which, one might argue, the patient voice (and those of patient advocates) have effectively become disembodied; pathways of care have radically changed and the scope for re-calibration seems ripe. The discussion of how a paradigm shift might be effected is, therefore, opportune.

This issue of the Future Healthcare Journal offers a multinational perspective and describes a wide variety of exemplary and inspiring initiatives. These initiatives recognise the need to alter complex systems and cultures that pose multiple challenges to achieving effective patient-powered safety, as well as recognising the considerable opportunities, on both sides.

One clear emerging theme (relevant to all, despite differing healthcare systems) is the lament that effective (national and comprehensive) strategies remain elusive. The approach is fragmented overall, and there is a clear and worrying undertone in the contributions that tokenism may, after all, win out.

This is a pressing and problematic conundrum. What will it take to change the discourse and bring about substantive organisational change?

There is probably no single answer. Effecting a genuine cultural shift, breaking down resistance and achieving buy-in from within will be tricky to achieve.

The doctor-patient relationship has traditionally been based on an unequal partnership of power and agency. Even having determined when and how to seek treatment (itself a complex decision-making process), becoming 'a patient' involves a certain amount of submission to professional 'expert' care (and corresponding loss of agency).

But patterns of illness have changed since the start of the NHS, from acute to chronic in which, for example, the heavy reliance on day-to-day self-management has meant that many patients have become well versed in understanding and dealing with their own conditions: 'experts' in their own right. Access to an abundance of medical information online is also underpinning a shift towards a more equitable patient partnership model. It is clear, too, that advocacy is a driver for patient safety. Yet arcane NHS practices and processes still fail to recognise (and capitalise) on this adequately.

At the same time, and this is well observed in Bucknall et al's paper, there is a continuum of patient engagement from passive to active that makes a 'one size fits all' solution impracticable. ${ }^{2}$ Navigating the system is hard for many (including families and carers). Not all patients are engaged with their health, many structural constraints interfere with this. There are numerous barriers to making one's voice heard. And not everyone aspires to be the 'expert patient'. Add to that, the sheer difficulty of being forceful or coherent when feeling vulnerable and unwell simply cannot be over-estimated.

Carefully constructed checklists may be a start towards a more equitable dialogue, but will suit some situations (and patients) better than others. ${ }^{3}$ While a tick-box/toolkit approach is a step forward (and iterative adjustment should result in continual improvement), it cannot readily resolve more nuanced scenarios, which are heavily reliant on mutually respectful and close communication between patient (or advocate/carer) and healthcare professionals. The latter requires a fundamental re-set and, in a ward setting, it may be that nursing staff are key.

Legislative changes, such as the introduction of 'Ryan's Rule' in Queensland, Australia, in which families and friends have the right to request an urgent independent review if they have concerns, could be part but not all of an answer. ${ }^{2}$ Would enshrining patient powered safety through policy be a useful catalyst?

There is little discussion of comorbidity and multimorbidity, yet there are clear issues in which long-term health conditions (such as diabetes) suffer when someone is admitted for another reason (including people with long-term health conditions being prevented from self-medicating during their hospital stay).

Additionally, there is the ongoing challenge of and lessons learned from the COVID-19 pandemic, including the backlog caused by the pandemic catapulting some clinicians into sudden and vastly increased workloads. This carries with it the risk of serious mistakes inevitably increasing under enormous, even overwhelming, pressure and the potentially tragic aftermath of that for clinicians and patients and their families. Several contributions focus on systems-based safety and performance initiatives designed to reduce the chance of such mistakes happening, thereby protecting the wellbeing of the workforce, sometimes drawing on the experience of other industries with similar risk-based profiles in order do to so. ${ }^{2,4-6}$ This is surely to be welcomed.

Lastly, while it is understandable that the focus is largely on patient safety in a hospital setting, this is reductive from a patient perspective. It reinforces the silo mentality that still prevails in the NHS. Patient safety is ultimately an issue that extends beyond boundaries of the hospital ward, the clinic or the general practice surgery. More discussion is needed on the parameters of patient safety, including appropriate support 'beyond the clinic', together with the pressing need to tackle health inequalities and the realisation of properly integrated care.

\section{Sarah Spain Editorial board member}

\section{References}

1 Subbe CP, Ahsan S, Smith L, Frey Renggli J. An audible patient voice: How can we ensure that patients are treated as partners in their own safety? FHJ 2021;8:e564-66.

2 Bucknall T, Quinney R, Booth L et al. When patients (and families) raise the alarm: Patient- and family-activated rapid response as a safety strategy for hospitals. FHJ 2021;8:e609-12.

3 Harris K, Russ S. Patient-centred safety checklists as an empowerment tool for patient involvement in patient safety: concepts, considerations and recommendations. FHJ 2021;8:e567-73.

4 Sujan M, Pickup L, Bowie P et al. The contribution of human factors and ergonomics to the design and delivery of safe future healthcare. FHJ 2021;8:e574-79.

5 Weaver S, Stewart K, Kay L. Systems-based investigation of patient safety incidents. FHJ 2021;8:e593-97.

6 O'Brien N, Durkin M, Lachman P. Lessons post-COVID-19 from national and international approaches to safety and quality in healthcare. FHJ 2021;8:e602-8. 